Article

\title{
Lyophilised Platelet-Rich Fibrin: Physical and Biological Characterisation
}

\author{
Nurul Aida Ngah ${ }^{1,2, *(\mathbb{D})}$, George J. Dias ${ }^{3}$, Darryl C. Tong ${ }^{1}$, Siti Noor Fazliah Mohd Noor ${ }^{4}(\mathbb{D}$, \\ Jithendra Ratnayake ${ }^{1} \mathbb{D}$, Paul R. Cooper ${ }^{1}$ and Haizal Mohd Hussaini ${ }^{1,5}$ \\ 1 Faculty of Dentistry, Sir John Walsh Research Institute, University of Otago, P.O. Box 56, \\ Dunedin 9054, New Zealand; darryl.tong@otago.ac.nz (D.C.T.); jithendra.ratnayake@otago.ac.nz (J.R.); \\ p.cooper@otago.ac.nz (P.R.C.); haizal.mh@otago.ac.nz (H.M.H.) \\ 2 Faculty of Dentistry, Universiti Teknologi MARA, Sungai Buloh Campus, Jalan Hospital, \\ Sungai Buloh 47000, Malaysia \\ 3 Department of Anatomy, School of Biomedical Sciences, University of Otago, P.O. Box 56, \\ Dunedin 9054, New Zealand; george.dias@otago.ac.nz \\ 4 Craniofacial and Biomaterial Sciences Cluster, Advanced Medical and Dental Institute, Universiti Sains \\ Malaysia, Kepala Batas 13200, Malaysia; fazliah@usm.my \\ 5 Faculty of Dental Medicine, Kampus A Universitas Airlangga, Surabaya 60132, Indonesia \\ * Correspondence: dr.aidangah@uitm.edu.my
}

check for updates

Citation: Ngah, N.A.; Dias, G.J.; Tong, D.C.; Mohd Noor, S.N.F.; Ratnayake, J.; Cooper, P.R.; Hussaini, H.M. Lyophilised Platelet-Rich Fibrin: Physical and Biological Characterisation. Molecules 2021, 26, 7131. https:// doi.org/10.3390/molecules26237131

Academic Editor: Toru Ogasawara

Received: 29 October 2021

Accepted: 23 November 2021

Published: 25 November 2021

Publisher's Note: MDPI stays neutral with regard to jurisdictional claims in published maps and institutional affiliations.

Copyright: (c) 2021 by the authors. Licensee MDPI, Basel, Switzerland. This article is an open access article distributed under the terms and conditions of the Creative Commons Attribution (CC BY) license (https:// creativecommons.org/licenses/by/ $4.0 /)$.

\begin{abstract}
Background: Platelet-rich fibrin (PRF) has gained popularity in craniofacial surgery, as it provides an excellent reservoir of autologous growth factors (GFs) that are essential for bone regeneration. However, the low elastic modulus, short-term clinical application, poor storage potential and limitations in emergency therapy use restrict its more widespread clinical application. This study fabricates lyophilised PRF (Ly-PRF), evaluates its physical and biological properties, and explores its application for craniofacial tissue engineering purposes. Material and methods: A lyophilisation method was applied, and the outcome was evaluated and compared with traditionally prepared PRF. We investigated how lyophilisation affected PRF's physical characteristics and biological properties by determining: (1) the physical and morphological architecture of Ly-PRF using SEM, and (2) the kinetic release of PDGF-AB using ELISA. Results: Ly-PRF exhibited a dense and homogeneous interconnected 3D fibrin network. Moreover, clusters of morphologically consistent cells of platelets and leukocytes were apparent within Ly-PRF, along with evidence of PDGF-AB release in accordance with previously reports. Conclusions: The protocol established in this study for Ly-PRF preparation demonstrated versatility, and provides a biomaterial with growth factor release for potential use as a craniofacial bioscaffold.
\end{abstract}

Keywords: lyophilisation; platelet concentrate; platelet-rich fibrin; craniofacial regeneration; tissue engineering

\section{Introduction}

Platelet-rich fibrin (PRF) has gained popularity in craniofacial surgery and dentistry due to the absence of anticoagulant and xeno-origin components. It provides a rich reservoir of autologous growth factors (GFs) that are essential for bone regeneration, including (1) platelet-derived growth factors (PDGF), (2) transforming growth factor beta (TGF $\beta$ ), and (3) vascular endothelial growth factors (VEGF), with prolonged release of these GFs to surgical areas [1-3]. PRF was previously developed as fresh platelet concentrates for same-day application as an adjunct for regenerative bone therapies [4]. Its low elastic modulus, limited storage capabilities, and restrictions in emergency therapy use have constrained its more widespread clinical application [5-8].

A suitable method to preserve the unique properties of PRF (i.e., growth factor release) and its microarchitecture is required to optimise its clinical application [9]. The search for new approaches to preserve the biological function of platelets is considerably important; 
thus, lyophilisation or a freeze-drying method was proposed to address the current drawbacks of the short clinical half-life of fresh platelet concentrates [10]. In this context, PRF lyophilisation, i.e., freezing followed by water sublimation and the subsequent removal of water vapour, was proposed as a consistent method for fabrication for an off-the-shelf product with improved stability, ready for future application [10]. Lyophilised or freeze-dried PRF (Ly-PRF) can be prepared on an autologous basis, and can be applied for treatments requiring multiple applications, such as alveolar cleft bone grafting. The lyophilisation of PRF was previously found to provide better storage stability, with a longer half-life and preservation of GFs [11]. Indeed, recent studies showed that there is justification for the development of large-scale production of Ly-PRF for future usage, particularly for bone augmentation $[12,13]$. There is a continuous release of platelet-derived growth factor $A B$ (PDGF-AB) from fresh PRF. However, little is known about the preservation and release pattern of PDGF-AB in Ly-PRF, and its three-dimensional (3D) microarchitecture.

Consequently, this paper generates lyophilised PRF (Ly-PRF), characterises its physical and biological properties, and explores the potential application of the Ly-PRF in craniofacial tissue engineering by determining: (1) the physical and morphological architecture of Ly-PRF's using SEM, (2) the surface area and cross-sectional structure of Ly-PRF using SEM, and (3) the release kinetics of PDGF-AB using ELISA.

\section{Results}

\subsection{Demographic Data of Donors}

Five systemically healthy participants ( 2 males and 3 females) were enrolled. Their mean age was $22.6 \pm 1.14$ years. There were no health-related complications reported prior to, during, or after blood collection.

\subsection{Morphological Analysis of Ly-PRF}

The mean weight of PRF and Ly-PRF from the five donor volunteers is shown in Table 1. After the freeze-drying process, the Ly-PRF material resembled a sponge, with a combination of yellowish and red colouration this was consistent in structure with previous protocols [3,14]. The middle layer was carefully removed using forceps along with a thin portion of the RBC layer, and this represents the traditional PRF fraction. This material had gel-like consistency (Figure 1A,B). The isolated PRF had a minimal weight of $970 \pm 0.56 \mathrm{mg}$ and a maximal weight of $1675 \pm 0.24 \mathrm{mg}$. The lowest weight for Ly-PRF was $104 \pm 0.03 \mathrm{mg}$, and the highest weight was $367 \pm 0.12 \mathrm{mg}$. Figure 1C,D display the different morphology and physical characteristics of PRF and Ly-PRF. Weight loss ranged from 70\% to 91\% for all PRF samples following the freeze-drying process. The difference in PRF weight before and after the freeze-drying process was statistically significant $(p<0.05)$ (Table 2$)$. Figure 2 displays the physical appearance of Ly-PRF before and after being ground into granules using a mortar and pestle.

Table 1. Comparative weight of PRF and Ly-PRF.

\begin{tabular}{ccc}
\hline Donor Samples & $\begin{array}{c}\text { PRF (mg) } \\
\text { (Mean } \pm \text { SD) }\end{array}$ & $\begin{array}{c}\text { Ly-PRF (mg) } \\
\text { (Mean } \pm \text { SD) }\end{array}$ \\
\hline 1 & $970 \pm 0.56$ & $284 \pm 0.32$ \\
2 & $1675 \pm 0.24$ & $367 \pm 0.12$ \\
3 & $1153 \pm 0.55$ & $104 \pm 0.03$ \\
4 & $1295 \pm 0.39$ & $281 \pm 0.15$ \\
5 & $1234 \pm 0.68$ & $274 \pm 0.24$ \\
\hline
\end{tabular}



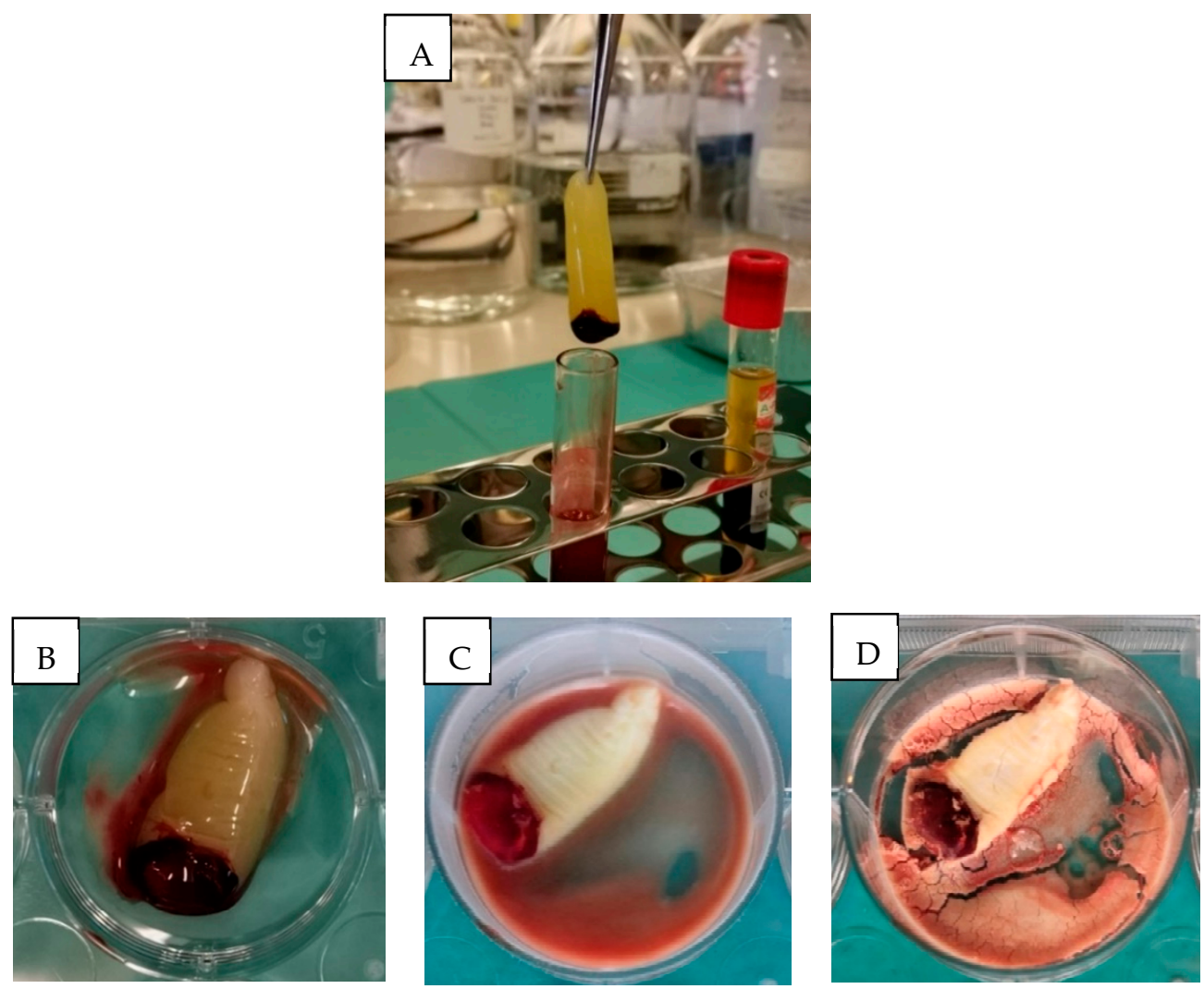

Figure 1. Platelet-rich fibrin (PRF) preparation. (A) Venous blood centrifuged for separation. PRF clot formed in the tube centre between red blood cells and platelet-poor plasma fractions. Appearance of (B) fresh PRF, (C) frozen PRF, and (D) lyophilised PRF.

Table 2. Comparative Ly-PRF pore sizes.

\begin{tabular}{cc}
\hline Donor Samples & Pore Sizes $(\boldsymbol{\mu m})($ Mean \pm SD) \\
\hline 1 & $30.91 \pm 24.44$ \\
2 & $41.16 \pm 33.80$ \\
3 & $17.34 \pm 23.05$ \\
\hline
\end{tabular}
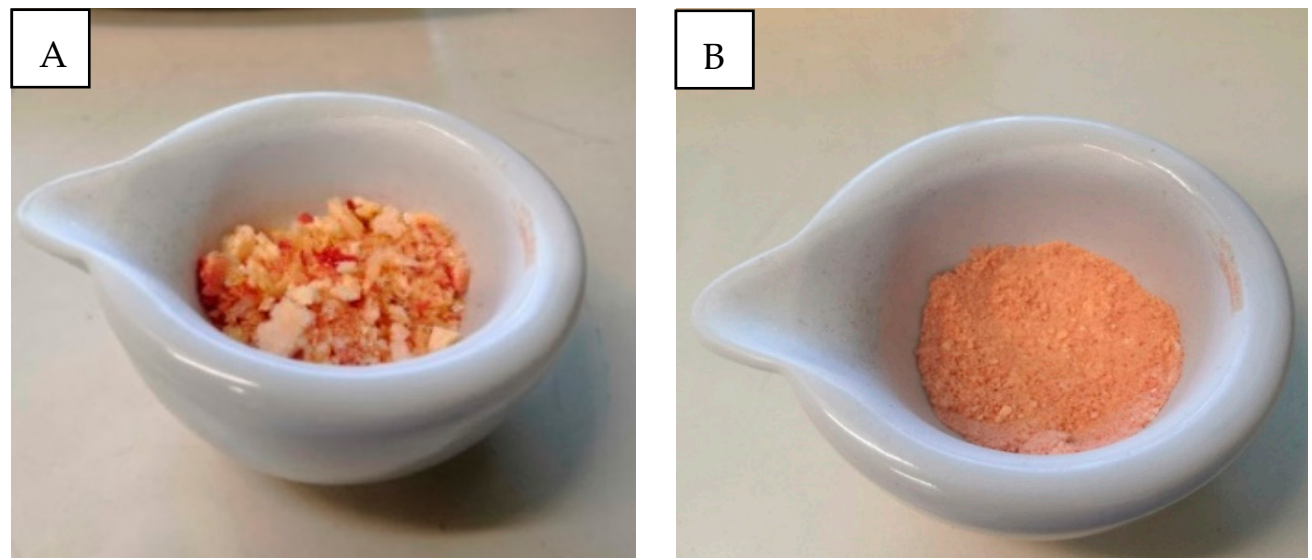

Figure 2. Physical appearance of lyophilised PRF. Ly-PRF (A) before and (B) after being ground into granules using a mortar and pestle. 
2.3. Structural and Surface Characterisation of Ly-PRF Using SEM

2.3.1. Surface Morphological Features of Ly-PRF

In general, under SEM, the surface morphology of Ly-PRF was irregular (Figure 3).
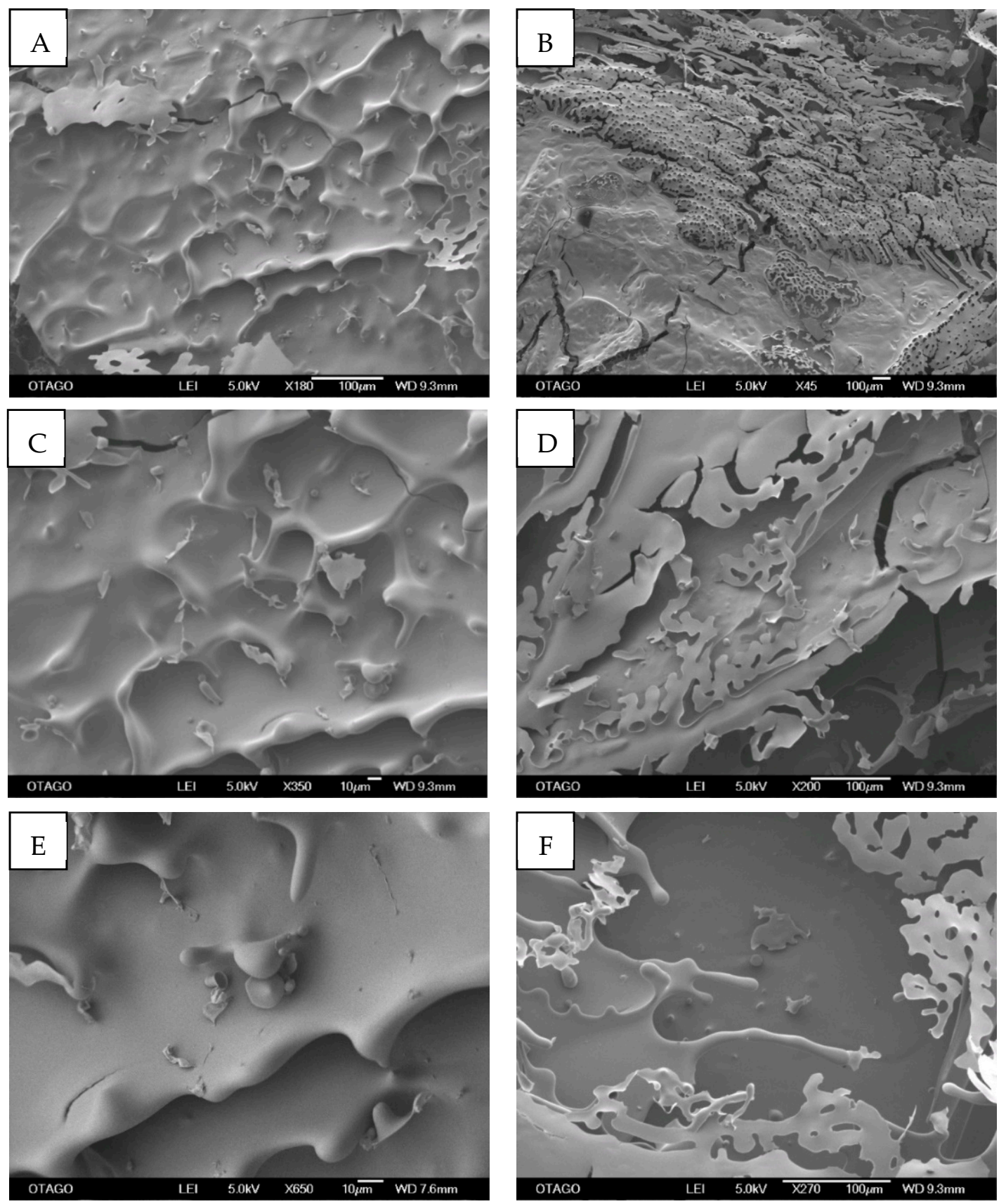

Figure 3. Representative scanning electron microscope (SEM) micrographs of surface of intact Ly-PRF, which showed irregular-surface topographical appearance. SEM magnifications: $((A) \times 45,(B) \times 180,(C) \times 200,(D) \times 270,(E) \times 350$, and (F) $\times 650)$. (Bar $=100 \mu \mathrm{m})$.

\subsubsection{Porous Structure of Ly-PRF}

The cross-sectional images of Ly-PRF exhibited porous microarchitecture with a heterogeneous network (Figure 4). The mean pore size observed for Ly-PRF was $30.91 \pm 24.44$, $41.16 \pm 33.80$ and $17.34 \pm 23.05$ for the three donors, respectively (Table 2). The pore size ranged from $2.45 \pm 23.05 \mu \mathrm{m}$ to $181.24 \pm 23.05 \mu \mathrm{m}$. Donor 3 exhibited the statistically significantly lowest average pore size among all donors $(p<0.05)$. 

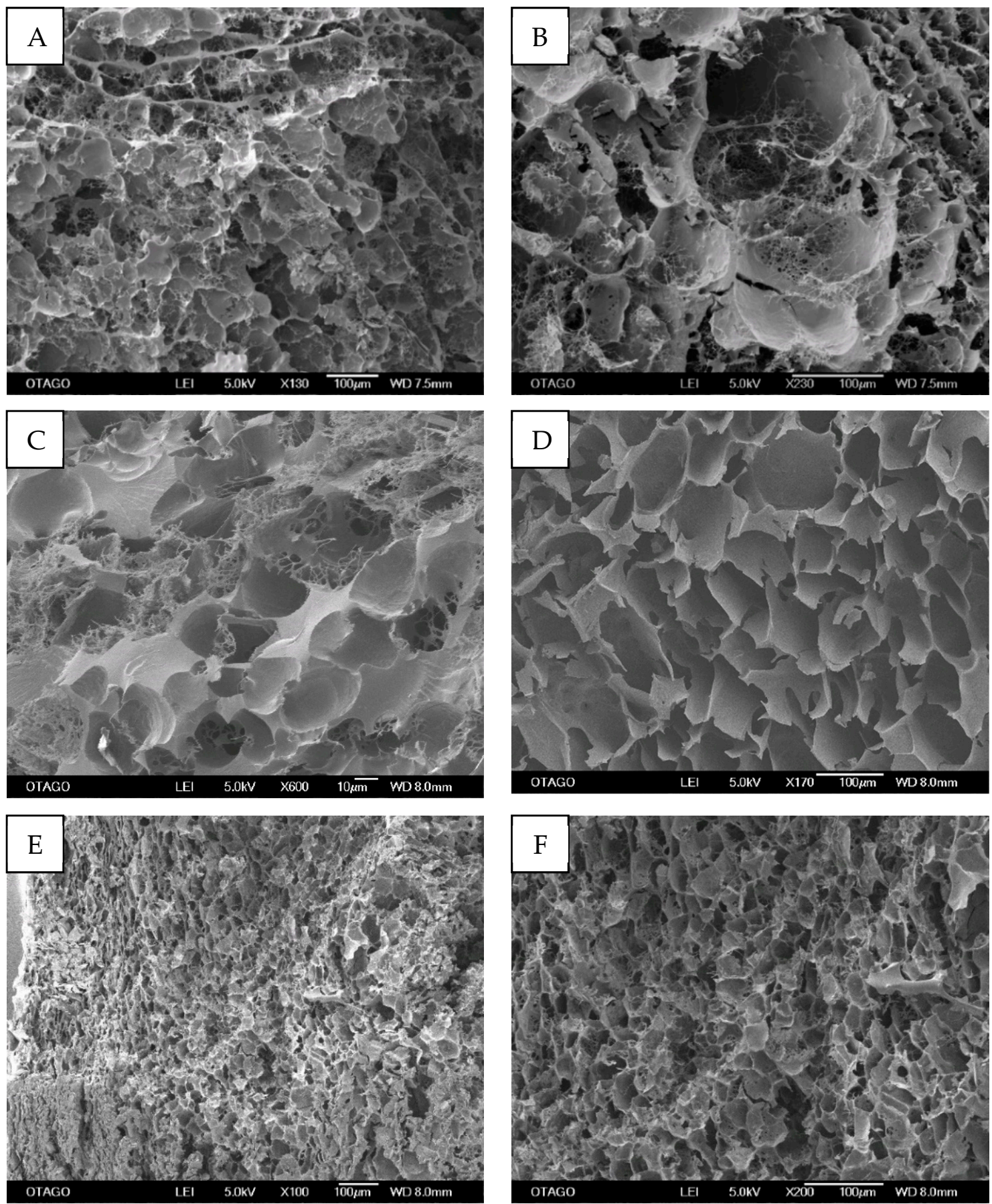

Figure 4. Cross-sections of intact Ly-PRF demonstrated a highly porous structure with a mixture of homogenous and heterogenous pore sizes. (SEM; original magnification: $(\mathbf{A}) \times 130,(\mathbf{B}) \times 230,(\mathbf{C}) \times 600,(\mathbf{D}) \times 170,(\mathbf{E}) \times 100$ and $(\mathbf{F}) \times 200)$. $($ Bar $=100 \mu \mathrm{m})$ Images $(\mathbf{A}, \mathbf{B})$ from Donor 1, images $(\mathbf{C}, \mathbf{D})$ from Donor 2 and images $(\mathbf{E}, \mathbf{F})$ from Donor 3.

\subsubsection{Fibrin Microarchitecture of Ly-PRF}

SEM images revealed a dense 3D fibrin framework within the Ly-PRF cross-sectional view (Figure 5). The mean diameter of the fibrin fibres was $339.70 \pm 133.20,384.10 \pm 151.50$, $332.70 \pm 133.20 \mathrm{~nm}$ for donor 1, donor 2 and donor 3, respectively (Table 3). Diameters ranged from $107 \pm 151 \mathrm{~nm}$ to $649 \pm 133.20 \mathrm{~nm}$. Donor 2 exhibited the statistically significant largest fibrin diameter of all donors $(p<0.05)$. 

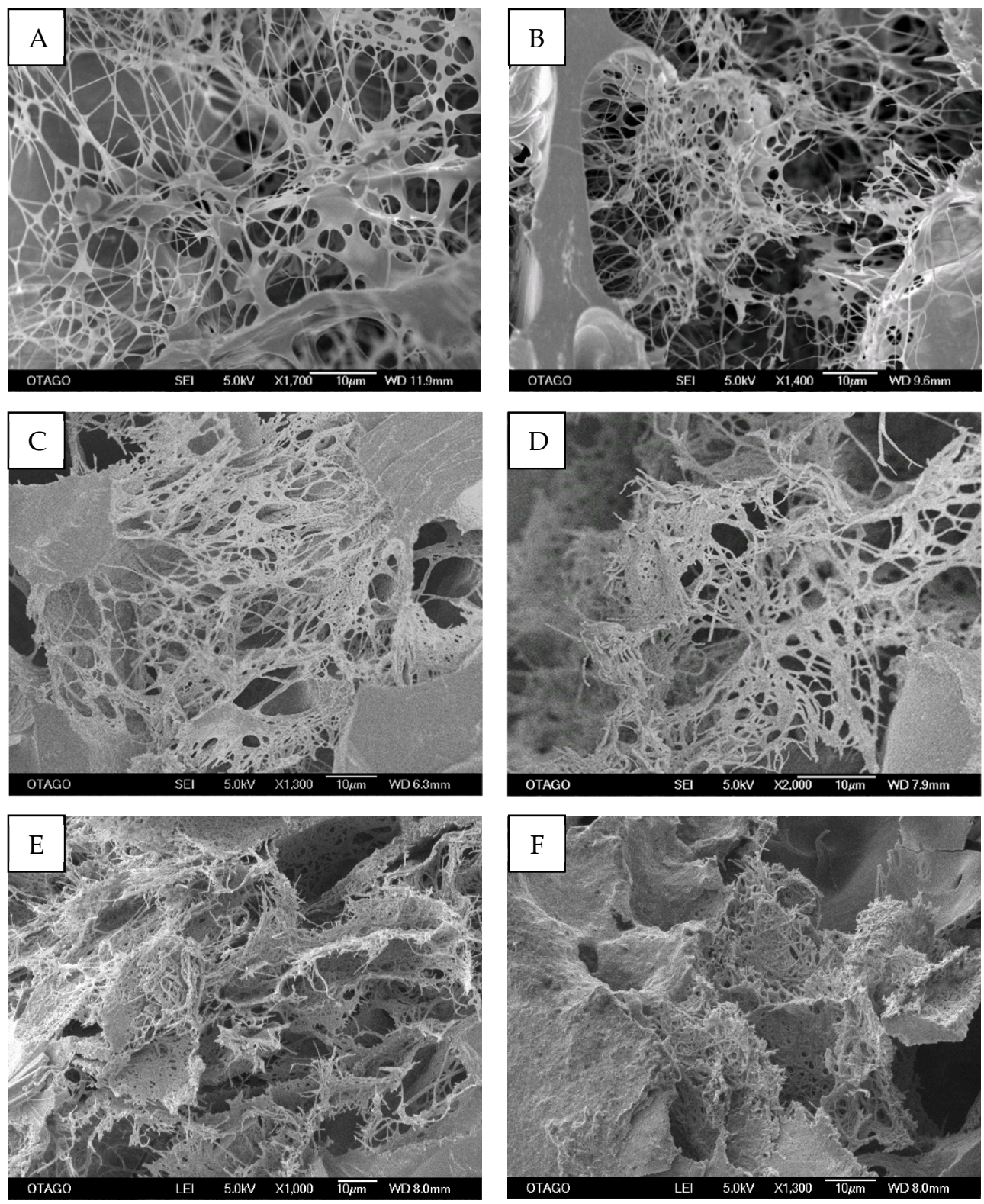

Figure 5. Highly dense and interconnected 3D fibrin microarchitecture in cross-sections of intact Ly-PRF. (SEM; original magnification: $(A) \times 1700,(B) \times 1400,(C) \times 1300,(D) \times 2000,(E) \times 1,000$ and (F) $\times 1300)$. (Bar $=10 \mu \mathrm{m})$. (A,B) Donor 1; (C,D) Donor 2; (E,F) Donor 3.

Table 3. Comparative Ly-PRF fibrin diameter.

\begin{tabular}{cc}
\hline Donor Samples & Fibrin $(\mathbf{n m})($ Mean \pm SD) \\
\hline 1 & $339.70 \pm 133.20$ \\
2 & $384.10 \pm 151.50$ \\
3 & $332.70 \pm 133.20$ \\
\hline
\end{tabular}

\subsubsection{Cell Entrapment within Ly-PRF}

Numerous cells were observed to be embedded in the complex three-dimensional network of the fibrin fibres. The majority of cells appeared as platelet and leukocyte clusters embedded within the fibrous structure, and were located at the border between the red and yellow clots of the Ly-PRF. Figure 6 demonstrates the clusters of platelets and leukocytes in the Ly-PRF. 

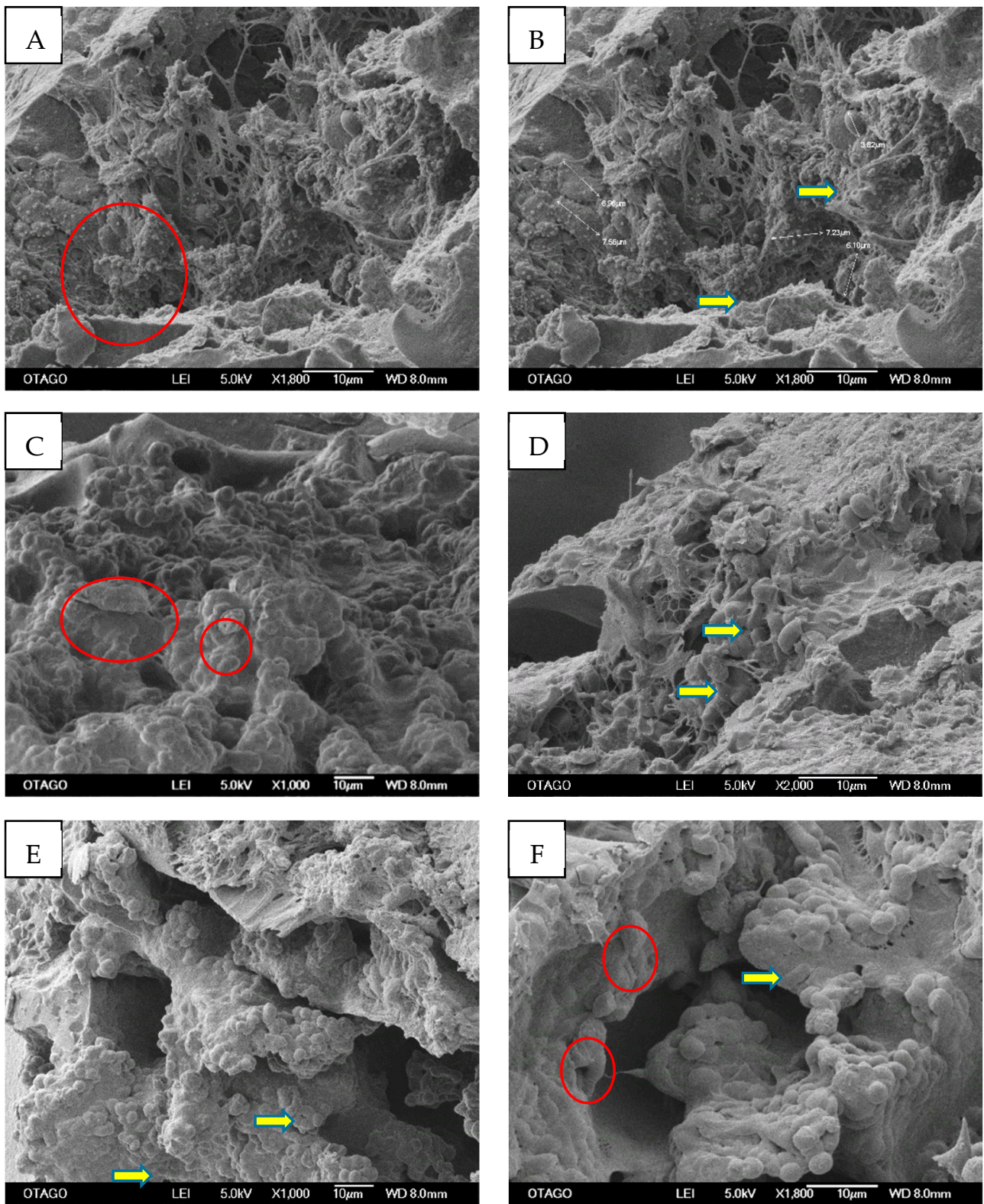

Figure 6. Representative SEM micrographs of cross-sections of intact Ly-PRF revealed entrapped clusters of platelets (yellow arrows) and leukocytes (red circles) within the Ly-PRF clot. (A) Representative image of a zone of enriched platelets with various degrees of activation. (SEM; original magnification: $(\mathbf{A}, \mathbf{B}) \times 1800,(\mathbf{C}) \times 1000,(\mathbf{D}) \times 2000,(\mathbf{E}) \times 1000$ and $(\mathbf{F}) \times 1800)$. $($ Bar $=10 \mu \mathrm{m})$.

\subsection{PDGF-AB ELISA}

The kinetic release of PDGF-AB growth factor in Ly-PRF was evaluated over 28 days using a commercially available enzyme-linked immunosorbent assay kit (Quantikinine ELISA (Human PDGF-AB), R\&D SYSTEMS ${ }^{\circledR}$, Minneapolis, MN, USA). This analysis was performed to assess the kinetic release pattern of PDGF-AB from the Ly-PRF. This timeframe was selected on the basis of previously reported studies that had performed similar analyses. The highest-level PDGF-AB growth factor release occurred on Day 1 $(71.09 \pm 41.24 \mathrm{pg} / \mathrm{mL})$, and the lowest release level was on Day $28(0.17 \pm 0.32 \mathrm{pg} / \mathrm{mL})$. A decreasing trend of PDGF-AB release was observed. Ly-PRF levels of PDGF-AB at Days 7, $10,14,21$, and 28 were statistically lower than that on Day $1(p<0.05)$. On Day 1, growth factor levels were 2-, 4-, 7-, 15-, and 426-fold statistically greater than those on Days 7, 10, 14,21 , and 28, respectively. No significant differences were observed in release after Day 
$10(p>0.05)$. The cumulative average release of PDGF-AB over 4 weeks was 94.08, 139.15, $418.66,156.55,254.43 \mathrm{pg} / \mathrm{mL}$, respectively (Figure 7). The PDGF-AB concentration was normalised using the relevant individual weight of Ly-PRF granules from each donor.

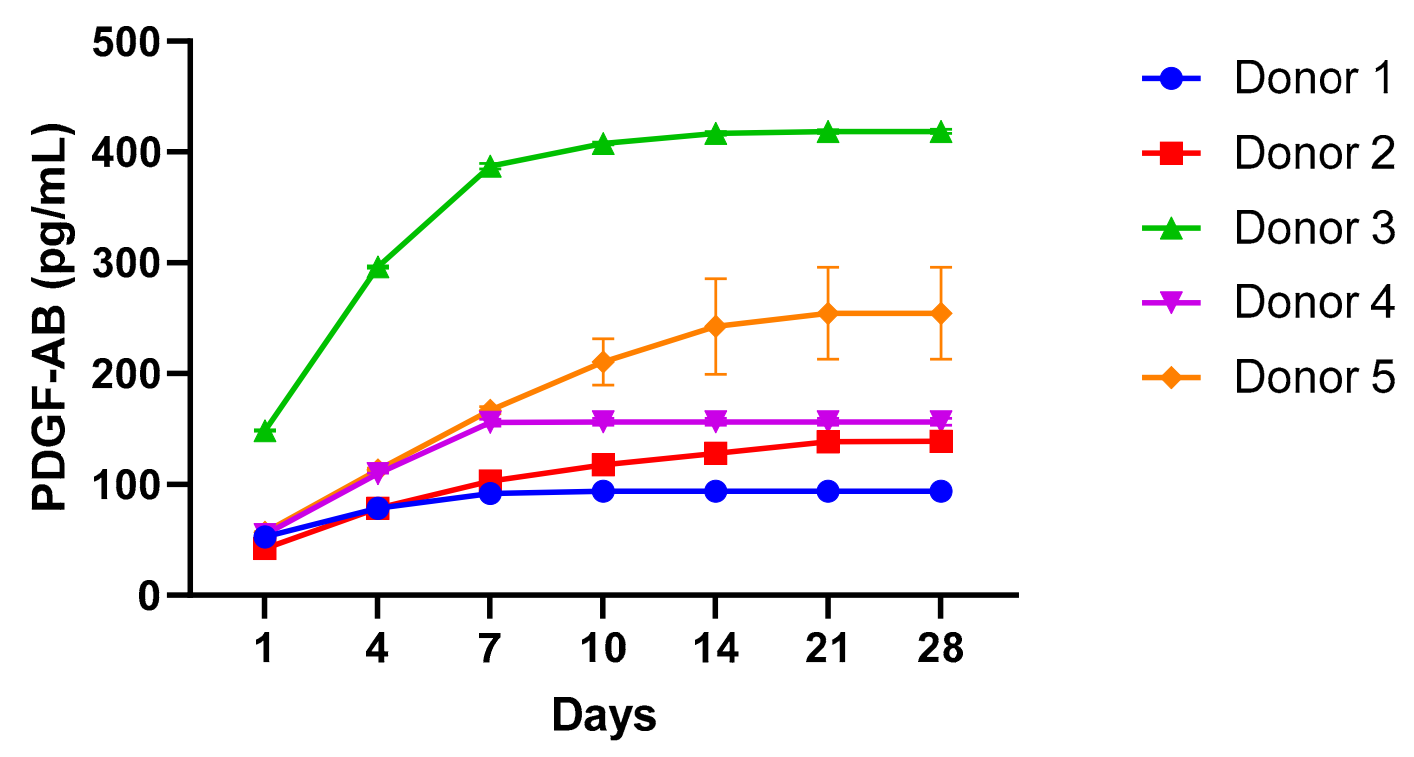

Figure 7. Cumulative kinetics for PDGF-AB growth factor release from five donors samples. Data presented as mean $\pm \mathrm{SD}$ of five biological replicates $(n=5)$ with 2 technical replicates $(n=2)$.

\section{Discussion}

The present study demonstrates the application of lyophilisation as a novel method for PRF preservation, primarily for maintaining the physical microarchitecture of PRF and preserving autologous PDGF-AB growth factor release. The Ly-PRF generated in this study displayed fundamental properties indicating its potential use as a bioactive scaffold and stimulant for bone regeneration.

\subsection{Ly-PRF Fabrication}

The Ly-PRF fabrication protocol developed for this study was based on the method previously described by Li et al. (2014), and Kardos et al. (2018) [8,15]. This method was primarily selected due to the successful fabrication and use of Ly-PRF as a biomaterial for cranial bone regeneration both in vitro and in vivo. This approach is the first to be reported in the literature for Ly-PRF fabrication, and similar protocols were deployed in this study for the purpose of standardisation. The physical characteristics of the Ly-PRF generated in this study exhibited spongelike appearance, similar to that previously reported for Ly-PRF in the literature $[8,15]$.

\subsection{Structural Microarchitecture of Ly-PRF}

The structural analysis of Ly-PRF, including the surface morphology and topography, fibrin network, cell attachment, and pore size, has thus far received limited attention. Hence, the current study provided a comprehensive description of Ly-PRF and hence contributes to a better understanding of this unique biomaterial.

\subsubsection{Surface Topography}

Our Ly-PRF exhibited relatively rugged and irregular surfaces. These data agree with previous studies that reported that Ly-PRF exhibited a compact structure with a rough surface texture [16]. Irregular topographies of bioactive materials are frequently reported as being advantageous for bone tissue engineering, since this property facilitates osteoblast adhesion, growth, and differentiation $[17,18]$. Thus, our Ly-PRF demonstrated surface 
roughness features and properties that likely enable bone regeneration when clinically placed.

\subsubsection{Fibrin Network}

Our results demonstrated that Ly-PRF exhibited a dense and homogeneous interconnected 3D fibrin network under SEM. The mean fibrin diameter identified in this study was larger than that reported by Bai et al. (2017), who had identified a maximal fibrin diameter of $140 \pm 39 \mathrm{~nm}$. This difference may have been due to their PRF being obtained from rabbit venous blood [19]. Fibrin plays a critical role in the three-dimensional structural stability of blood clots [20]. Previous studies suggested that the 3D network of the Ly-PRF is the main factor in providing the sustained release of growth factors from this unique biomaterial [21,22]. The prominent fibrin architecture of our Ly-PRF may have enabled a continuous and gradual release of growth factors throughout the study period. Furthermore, the unique property of the interconnected fibrin network structure in PRF may also protect growth factors from proteolysis. Hence, growth factors may sustain their function for a comparatively longer time period to enable their efficient promotion of bone regeneration [23]. Moreover, the solid walls around the pore (Figure 5) may have consisted of a highly polymerised and condensed fibrin matrix that surrounded the dense fibrin fibres. This structure arises due to the mechanical centrifugal and mild compressive forces that are generated during the application of Choukroun's PRF protocol. A similar structure was previously reported by Dohan Ehrenfest et al. (2010) [14].

\subsubsection{Cell Distribution}

Clusters of morphologically consistent cells of platelets and leukocytes were identified within the Ly-PRF in concordance with previous reports [20]. The majority of identified cells were morphologically consistent with platelets and leukocyte clusters, and were located between the junction of the Ly-PRF and the red blood clot. This finding agrees with previous studies that reported a maximal platelet/leukocyte density apparent within the first millimetre of the yellow clot, immediately adjacent to the red blood clot fraction [14,24]. These studies highlighted the importance of having a thorough understanding of PRF clot design in order to obtain optimal clinical outcomes when using this material. Thus, it is crucial to maintain a minimal red blood clot layer at the PRF clot end location in order to obtain the highest number of platelets and leukocytes. Platelet distribution in PRF matrices is heavily influenced by centrifugal force, rotor type, and PRF fabrication protocols [25]. Leukocytes contribute significantly to PRF's antibacterial properties and are a distinctive feature of the material, which is why it is also referred to as leukocyte-and platelet-rich fibrin (LPRF). This terminology emphasises the importance of leukocytes and indicates their significance to PRF success for stimulating bone regeneration [22,24,26,27].

\subsubsection{Porosity}

Ly-PRF is a highly porous material with an average pore size diameter of $29.80 \pm 27 \mu \mathrm{m}$. The mean pore sizes identified in our study were larger than those previously reported $[8,15]$. $\mathrm{Li}$ et al. (2014) observed that the mean diameter of the Ly-PRF bioscaffold used in their research for craniofacial regeneration was $8.06 \pm 0.31 \mu \mathrm{m}$ [7]. Kardos et al. (2018) reported that their Ly-PRF possessed a more compact structure with considerably fewer pores than that of $\mathrm{Li}$ et al. (2014). The larger mean pore diameter detected in our investigation may have been due to the mean of multiple series of Ly-PRF sections performed along the length of the Ly-PRF. Pore diameters varied according to the sectioned area. This agreed with a previous study that indicated that the area in closest proximity to the red blood cell (RBC) layer exhibited a more compact structure compared with the plasma layer [19].

An earlier study also reported that Ly-PRF was nearly twice as effective as fresh PRF at stimulating mineralisation. This beneficial property was attributed to the increased pore size in the Ly-PRF, which was 13 times greater than that found in fresh PRF [8]. Additionally, increased pore size enhances tissue engineering features, including promoting 
cell adhesion, proliferation, and tissue-repair-related gene expression. Therefore, our data support the notion that the lyophilisation process preserves PRF's microarchitecture while simultaneously enhancing its tissue engineering properties, such as increased pore diameter.

\subsection{Growth Factors}

Transforming growth factors beta-1 (TGF- $\beta 1$ ), platelet-derived growth factors (PDGF), and vascular endothelial growth factors (VEGF) are three major growth factors present in PRF $[9,22]$. This study focused primarily on PDGF-AB, as it is one of the most highly released growth factors from PRF. PDGF-AB promotes tissue healing by accelerating collagen production, hence increasing wound strength and initiating callus development [20]. Additionally, it can attract and stimulate bone progenitor cells, which is critical for bone regenerative therapies in maxillofacial and craniofacial surgery $[28,29]$. During the early stages of healing, PDGF-AB attracts inflammatory cells, such as macrophages and mesenchymal stem cells (MSCs), to the injury site [30]. It can also stimulate local cell proliferation, differentiation, and extracellular matrix formation [21,27,31]. In our study, we demonstrated the presence and sustained release of the PDGF-AB in Ly-PRF following a freeze-drying process. PDGF is resilient to extreme heat, $\mathrm{pH}$, and several proteases due to the presence of 8 cysteine residues per chain in disulphide bonds [30]. Our data demonstrated a similar pattern of PDGF-AB release from Ly-PRF in our five donor samples over 28 days. Our results also indicated that there was a gradual release from the onset of the study, which subsequently plateaued over time. This kinetic release pattern agrees with a recent study of Ly-PRF $[9,32]$. However, our overall PDGF-AB quantities were lower than those previously reported [32]. These data indicate that the complexity and heterogeneity of this biomaterial significantly varies between individuals.

The main issue concerning platelet concentrate technologies is not the quantity of growth factors present but rather the cells, 3D fibrin network, and growth factor types that are integrated into the final product. Furthermore, the prolonged release of growth factors enables a more effective bone repair process. The importance of PDGF-AB in the early stages of bone regeneration was highlighted [33]. Ly-PRFs may be favourable for bone regeneration, where Ly-PRF can be used as an autologous growth factor reservoir or as a non-weight-bearing autologous bioscaffold within craniofacial tissue.

\subsection{Ly-PRF Tissue Bank Potential and Future Recommendations}

Improvements in medical technologies have resulted in greater quantities and a broader range of tissue banking being available in order to enhance patients' quality of life [34]. The potential benefit of the tissue bank for platelet concentrate allografts was proven in vivo, and in clinical trials in plastic and reconstructive surgery applications. This technique is appropriate for the treatment of chronic wounds $[35,36]$. The combination of tissue-banking and tissue-engineering technologies to develop biomaterials for regenerative therapy has an ever-increasing chance of success [37]. Ly-PRF exhibited many of the necessary key characteristics for a biomaterial for tissue-engineering purposes. Therefore, future research should explore other relevant properties of this biomaterial as a potential source for tissue bank platelet concentrate.

Indeed, Ly-PRF displayed significant versatility as a promising biomaterial for craniofacial regeneration. The presence and persistence of PDGF-AB release in Ly-PRF were demonstrated, and its high porosity and interconnected fibrin network indicate its potential as a bone scaffold. Furthermore, as it is naturally derived, it can provide an effective and personalised approach for tissue repair that can be archived for future use [38,39]. The mean pore size of $29.80 \pm 27 \mu \mathrm{m}$ and degradability of Ly-PRF make it less suited for use as a craniofacial scaffold on its own. However, in combination with collagen-chitosan membranes, poly-lactic-co-glycolic acid (PLGA) with nanohydroxyapatite and polyvinyl alcohol hydrogel scaffolds, its application may be significantly enhanced for bone regeneration $[7,9,38]$. Consequently, further research to determine the physicochemical and 
biological characteristics of composite biomaterials that incorporate Ly-PRF are necessary to optimise it for clinical application.

\subsection{Study Limitations}

A limitation of this study is that we focused primarily on PDFG-AB. Therefore, future research should emphasise additional GFs present in the LyPRF, including PDGF-AA, PDGF-BB, TGF-B1, and VEGF. Furthermore, future studies should explore the quantity and viability of the cellular content (particularly platelet and leukocyte) to determine its role in bone regeneration and antibacterial activity of the LyPRF. Nevertheless, additional research is required to ascertain if there is a need for the sterilisation of this material prior to clinical application.

\section{Materials and Methods}

\subsection{Ethical Approval and Māori Consultation}

Ethical approval was obtained from the University of Otago Human Ethics Committee (Health) (approval number H19/057) and the study was conducted in full accordance with the World Medical Declaration of Helsinki. Consultation with the Māori was undertaken with the Ngāi Tahu Research Consultation Committee.

\subsection{Subjects Selection}

PRF was prepared from human venous blood obtained from five healthy volunteers [23]. All subjects enrolled in this research gave informed consent, and were provided with an explanation of the research protocol and the expected benefits from this study, and future potential clinical use by the community. Inclusion criteria for this study were: age range from 25 to 35 years old, being fit and healthy, nonsmokers, and with no history of recent aspirin intake or anticoagulant drugs. Participants who had recently taken antiplatelet or anticoagulant medication, had any chronic medical issues, or were smokers were excluded from the study.

\subsection{Fabrication of Lyophilised Platelet-Rich Fibrin}

Venous blood was taken without anticoagulant in a $10 \mathrm{~mL}$ glass tube (A-PRF' ${ }^{\mathrm{TM}}$ by Choukroun, Auckland, New Zealand) and was immediately centrifuged according to Choukroun's protocol. Briefly, the blood was centrifuged at $400 \times g$ (approximately 3000 $\mathrm{rpm}$ ) for $10 \mathrm{~min}$ using an Eppendorf Centrifuge $5810 \mathrm{R}$ [14,16]. Following centrifugation, the blood separated into three layers distinguished by the following: (1) a transparent and acellular layer characterised the upper layer, (2) the middle layer was the PRF (fibrin buffy coat) layer, and (3) the third layer represented red blood cells. The middle layer was removed from the centrifuge tube using sterile forceps, and separated from the third layer using sterile scissors with a thin layer of RBC being retained. Freshly prepared PRF matrices were carefully compressed using a stainless-steel PRF compression device obtained from A-PRF kit (Ostralos Ltd., Auckland, New Zealand). To prepare Ly-PRF, intact fresh PRF was frozen and stored at $-80^{\circ} \mathrm{C}$ for 30 min before being freeze-dried (FreeZone ${ }^{\circledR}$ Triad Freeze Dry System Models 740000 Series, Labconco, Kansas City, MO, USA) overnight at $-51^{\circ} \mathrm{C}$ to produce Ly-PRF $[8,15]$. Samples were ground into granules using a mortar and pestle before storage at $4{ }^{\circ} \mathrm{C}[7,9,40]$.

\subsection{Quantification of Growth Factors in Lyophilised PRF}

PDGF-AB was selected as a representative molecule to quantify the release of growth factors from the fabricated Ly-PRF. The weight of Ly-PRF granules was based on the mean weight $(\mathrm{mg})$ of each intact Ly-PRF from each donor [41]. Ly-PRF granules were incubated in $4 \mathrm{~mL}$ of Dulbecco's Modified Eagle's Medium (DMEM) in a cell culture flask at $37^{\circ} \mathrm{C}$ and with $5 \% \mathrm{CO}_{2}$ for 28 days to evaluate the kinetics of PDGF-AB growth factor release. We collected $4 \mathrm{~mL}$ of conditioned medium at a range of time-points (Days 1, 4, 7, 14, 21, and 28), which was replaced with an equal volume of DMEM [21,32,41]. Prior to using an 
enzyme-linked immunosorbent assay (ELISA) kit (Quantikine ELISA (Human PDGF-AB), R \& D SYSTEMS ${ }^{\circledR}$, Minneapolis, MN, USA), samples were stored at $-80^{\circ} \mathrm{C}$. All reagents, working standards, sample preparation, and growth factor quantification were performed according to manufacturer instructions. Results are presented as the mean \pm standard deviation and were analysed for statistically significant differences [7].

\subsection{Scanning Electron Microscopy (SEM) Analysis}

SEM analysis was conducted to characterise the fabricated Ly-PRF microarchitecture. SEM enabled the evaluation of the materials' surface, fibrin distribution, pore sizes assessment and cell entrapment within the fibrin network. It was also used to evaluate the overall surface topography and cross-sectional architectures of Ly-PRF. After $1 \mathrm{~h}$ of fixation in $2.5 \%$ glutaraldehyde (G6257-Sigma-Aldrich, St. Louis, MO, USA), Ly-PRFs were dehydrated using graded ethanol [14,42]. The Ly-PRF was gold-sputter-coated before being observed by SEM (JOEL, JSM-6700F, Tokyo, Japan), as shown in Figure 3. Fibre and pore sizes were obtained by measuring at least 100 fibres and pores, respectively, from three distinctive images using ImageJ software (Bethesda, MD, USA) to obtain their mean diameter and pore sizes [31].

\subsection{Statistical Analysis}

All statistical analyses were conducted using statistical analysis software PRISM (GraphPad Prism 6, San Diego, CA, USA). Data for the experiments are expressed as mean \pm standard deviation (SD). Each experiment was performed at least twice and prepared (n $=3$ ) for each volunteer at designated intervals. Repeated-measures within-group ANOVA was performed to compare the concentration of the PDGF-AB concentration at a 7 timepoint measurement within the 28 days. Comparison for PDGF-AB concentration was performed using repeated-measures between-group ANOVA (two-way) with Tukey's post hoc correction for multiple comparisons being undertaken. Moreover, the comparison of pore sizes and fibrin diameters between the three donors were conducted using ANOVA (one-way) with Turkey's post hoc test performed for multiple comparison. A $p$ value $<0.05$ was considered to be statistically significant.

\section{Conclusions}

The protocol established in this study for generating Ly-PRF enables the production of a platelet concentrate with continuous growth factor release for bone regeneration applications in a simple, cost-effective, and organic approach. The Ly-PRF developed via the lyophilisation method demonstrated versatility as a potential biomaterial for use as a craniofacial bioscaffold. Apart from its unique properties as a reservoir for growth factor PDGF-AB, Ly-PRF exhibited fundamental scaffold properties. Future research should explore the properties of this material as a prospective source for the tissue banking of platelet concentrate.

Author Contributions: All authors contributed extensively to the work presented in this paper. Conceptualization, N.A.N. and H.M.H.; methodology, N.A.N., S.N.F.M.N. and D.C.T.; validation, N.A.N., S.N.F.M.N. and H.M.H.; formal analysis, N.A.N., S.N.F.M.N. and H.M.H.; resources, D.C.T., G.J.D., J.R., P.R.C. and H.M.H.; writing-original draft preparation, N.A.N., S.N.F.M.N. and H.M.H.; writing - review and editing, N.A.N., S.N.F.M.N., G.J.D., D.C.T., J.R., P.R.C. and H.M.H.; visualisation, N.A.N. and H.M.H.; supervision, H.M.H., S.N.F.M.N., D.C.T. and G.J.D. All authors have read and agreed to the published version of the manuscript.

Funding: Ministry of Higher Education, Malaysia and Universiti Teknologi MARA for scholarship funding of this project. This project was also financially funded by the NZ Dental Research Foundation (RF8.15 2020). We acknowledge the John Walsh Research Institute, Faculty of Dentistry, University of Otago for their support.

Institutional Review Board Statement: Not applicable. 
Informed Consent Statement: Informed consent was obtained from all subjects involved in the study. Ethical approval was obtained from the University of Otago Ethics Committee (Health) (H19/057).

Data Availability Statement: The data presented in this study are available on request from the corresponding author.

Acknowledgments: We would like to thank Liz Girvan for her expert help and assistance in SEM imaging, and Saifulizam Shadian for manuscript editing.

Conflicts of Interest: The authors declare no conflict of interest. The funders had no role in the design of the study; in the collection, analyses, or interpretation of data; in the writing of the manuscript, or in the decision to publish the results.

Sample Availability: Not applicable.

\section{References}

1. Choukroun, J.; Diss, A.; Simonpieri, A.; Girard, M.O.; Schoeffler, C.; Dohan, S.L.; Anthony, J.J.; Jaafar, M.; David, M. Platelet-rich fibrin (PRF): A second-generation platelet concentrate. Part IV: Clinical effects on tissue healing. Oral Surg. Oral Med. Oral Pathol. Oral Radiol. Endodontol. 2006, 101, e56-e60. [CrossRef]

2. Dohan, D.M.; Choukroun, J.; Diss, A.; Dohan, S.L.; Dohan, A.J.; Mouhyi, J.; Gogly, B. Platelet-rich fibrin (PRF): A secondgeneration platelet concentrate. Part II: Platelet-related biologic features. Oral Surg. Oral Med. Oral Pathol. Oral Radiol. Endodontol. 2006, 101, e45-e50. [CrossRef] [PubMed]

3. Miron, R.J.; Choukroun, J. Platelet Rich Fibrin in Regenerative Dentistry; John Wiley \& Sons Ltd.: Hoboken, NJ, USA, 2017.

4. Dohan Ehrenfest, D.M.; Diss, A.; Odin, G.; Doglioli, P.; Hippolyte, M.-P.; Charrier, J.-B. In vitro effects of Choukroun's PRF (platelet-rich fibrin) on human gingival fibroblasts, dermal prekeratinocytes, preadipocytes, and maxillofacial osteoblasts in primary cultures. Oral Surg. Oral Med. Oral Pathol. Oral Radiol. Endodontol. 2009, 108, 341-352. [CrossRef]

5. Ngah, N.A.; Ratnayake, J.; Cooper, P.R.; Dias, G.J.; Tong, D.C.; Mohd Noor, S.N.F.; Hussaini, H.M. Potential of Lyophilized Platelet Concentrates for Craniofacial Tissue Regenerative Therapies. Molecules 2021, 26, 517. [CrossRef] [PubMed]

6. Horimizu, M.; Kawase, T.; Nakajima, Y.; Okuda, K.; Nagata, M.; Wolff, L.F.; Yoshie, H. An improved freeze-dried PRP-coated biodegradable material suitable for connective tissue regenerative therapy. Cryobiology 2013, 66, 223-232. [CrossRef]

7. Xu, F.; Zou, D.; Dai, T.; Xu, H.; An, R.; Liu, Y.; Liu, B. Effects of incorporation of granule-lyophilised platelet-rich fibrin into polyvinyl alcohol hydrogel on wound healing. Sci. Rep. 2018, 8, 14042. [CrossRef] [PubMed]

8. Li, Q.; Reed, D.A.; Min, L.; Gopinathan, G.; Li, S.; Dangaria, S.J.; Li, L.; Geng, Y.; Galang, M.T.; Gajendrareddy, P.; et al. Lyophilized platelet-rich fibrin (PRF) promotes craniofacial bone regeneration through Runx2. Int. J. Mol. Sci. 2014, 15, 8509-8525. [CrossRef]

9. Zheng, L.; Wang, L.; Qin, J.; Sun, X.; Yang, T.; Ni, Y.; Zhou, Y. New Biodegradable Implant Material Containing Hydrogel with Growth Factors of Lyophilized PRF in Combination with an nHA/PLGA Scaffold. J. Hard Tissue Biol. 2015, 24, 54-60. [CrossRef]

10. Andia, I.; Perez-Valle, A.; Del Amo, C.; Maffulli, N. Freeze-Drying of Platelet-Rich Plasma: The Quest for Standardization. Int. J. Mol. Sci. 2020, 21, 6904. [CrossRef]

11. Nireesha, G.; Divya, L.; Sowmya, C.; Venkateshan, N.; Babu, M.; Lavakumar, V. Lyophilization/Freeze Drying-An Review. Int. J. Nov. Trends Pharm. Sci. 2013, 3, 87-98.

12. Nakatani, Y.; Agata, H.; Sumita, Y.; Koga, T.; Asahina, I. Efficacy of freeze-dried platelet-rich plasma in bone engineering. Arch. Oral Biol. 2017, 73, 172-178. [CrossRef] [PubMed]

13. Vocetkova, K.; Buzgo, M.; Sovkova, V.; Rampichova, M.; Staffa, A.; Filova, E.; Lukasova, V.; Doupnik, M.; Fiori, F.; Amler, E. A comparison of high throughput core-shell 2D electrospinning and 3D centrifugal spinning techniques to produce platelet lyophilisate-loaded fibrous scaffolds and their effects on skin cells. RSC Adv. 2017, 7, 53706-53719. [CrossRef]

14. Dohan Ehrenfest, D.M.; Del Corso, M.; Diss, A.; Mouhyi, J.; Charrier, J.-B. Three-Dimensional Architecture and Cell Composition of a Choukroun's Platelet-Rich Fibrin Clot and Membrane. J. Periodontol. 2010, 81, 546-555. [CrossRef] [PubMed]

15. Kardos, D.; Hornyak, I.; Simon, M.; Hinsenkamp, A.; Marschall, B.; Vardai, R.; Kallay-Menyhard, A.; Pinke, B.; Meszaros, L.; Kuten, O.; et al. Biological and Mechanical Properties of Platelet-Rich Fibrin Membranes after Thermal Manipulation and Preparation in a Single-Syringe Closed System. Int. J. Mol. Sci. 2018, 19, 3433. [CrossRef]

16. Dohan, D.M.; Choukroun, J.; Diss, A.; Dohan, S.L.; Dohan, A.J.; Mouhyi, J.; Gogly, B. Platelet-rich fibrin (PRF): A secondgeneration platelet concentrate. Part I: Technological concepts and evolution. Oral Surg. Oral Med. Oral Pathol. Oral Radiol. Endodontol. 2006, 101, e37-e44. [CrossRef]

17. Kamarajan, B.P.; Muthusamy, A.; Jagadeesh, K.A. Nano-rough topography of the scaffold enhances osteoblast attachment-A Review. Trends Biomater. Artif. Organs 2014, 28, 26-31.

18. Bruzauskaite, I.; Bironaite, D.; Bagdonas, E.; Bernotiene, E. Scaffolds and cells for tissue regeneration: Different scaffold pore sizes-different cell effects. Cytotechnology 2016, 68, 355-369. [CrossRef]

19. Bai, M.Y.; Wang, C.W.; Wang, J.Y.; Lin, M.F.; Chan, W.P. Three-dimensional structure and cytokine distribution of platelet-rich fibrin. Clinics 2017, 72, 116-124. [CrossRef]

20. Kang, Y.-H.; Jeon, S.H.; Park, J.-Y.; Chung, J.-H.; Choung, Y.-H.; Choung, H.-W.; Kim, E.-S.; Choung, P.-H. Platelet-Rich Fibrin is a Bioscaffold and Reservoir of Growth Factors for Tissue Regeneration. Tissue Eng. Part. A 2011, 17, 349-359. [CrossRef] 
21. He, L.; Lin, Y.; Hu, X.; Zhang, Y.; Wu, H. A comparative study of platelet-rich fibrin (PRF) and platelet-rich plasma (PRP) on the effect of proliferation and differentiation of rat osteoblasts in vitro. Oral Surg. Oral Med. Oral Pathol. Oral Radiol. Endodontol. 2009, 108, 707-713. [CrossRef]

22. Dohan Ehrenfest, D.M.; Bielecki, T.; Jimbo, R.; Barbé, G.; Del Corso, M.; Inchingolo, F.; Sammartino, G. Do the fibrin architecture and leukocyte content influence the growth factor release of platelet concentrates? An evidence-based answer comparing a pure platelet-rich plasma (P-PRP) gel and a leukocyte- and platelet-rich fibrin (L-PRF). Curr. Pharm. Biotechnol. 2012, 13, 1145-1152. [CrossRef]

23. Lundquist, R.; Dziegiel, M.H.; Agren, M.S. Bioactivity and stability of endogenous fibrogenic factors in platelet-rich fibrin. Wound Repair Regen. 2008, 16, 356-363. [CrossRef]

24. Wang, Z.; Mudalal, M.; Sun, Y.; Liu, Y.; Wang, J.; Wang, Y.; SUN, X.; Zhou, Y. The Effects of Leukocyte-Platelet Rich Fibrin (L-PRF) on Suppression of the Expressions of the Pro-Inflammatory Cytokines, and Proliferation of Schwann Cell, and Neurotrophic Factors. Sci. Rep. 2020, 10, 2421. [CrossRef] [PubMed]

25. Aizawa, H.; Tsujino, T.; Watanabe, T.; Isobe, K.; Kitamura, Y.; Sato, A.; Yamaguchi, S.; Okudera, H.; Okuda, K.; Kawase, T. Quantitative Near-Infrared Imaging of Platelets in Platelet-Rich Fibrin (PRF) Matrices: Comparative Analysis of Bio-PRF, Leukocyte-Rich PRF, Advanced-PRF and Concentrated Growth Factors. Int. J. Mol. Sci. 2020, 21, 4426. [CrossRef] [PubMed]

26. Jasmine, S.; Thangavelu, A.; Janarthanan, K.; Krishnamoorthy, R.; Alshatwi, A.A. Antimicrobial and antibiofilm potential of injectable platelet rich fibrin-A second-generation platelet concentrate-Against biofilm producing oral staphylococcus isolates. Saudi J. Biol. Sci. 2020, 27, 41-46. [CrossRef]

27. Wirz, S.; Dietrich, M.; Flanagan, T.C.; Bokermann, G.; Wagner, W.; Schmitz-Rode, T.; Jockenhoevel, S. Influence of platelet-derived growth factor-AB on tissue development in autologous platelet-rich plasma gels. Tissue Eng. Part. A 2011, 17, 1891-1899. [CrossRef]

28. Gaßling, V.L.W.; Açil, Y.; Springer, I.N.; Hubert, N.; Wiltfang, J. Platelet-rich Plasma and Platelet-rich fibrin in human cell culture. Oral Surg. Oral Med. Oral Pathol. Oral Radiol. Endodontol. 2009, 108, 48-55. [CrossRef]

29. Mohan, S.P.; Jaishangar, N.; Devy, S.; Narayanan, A.; Cherian, D.; Madhavan, S.S. Platelet-Rich Plasma and Platelet-Rich Fibrin in Periodontal Regeneration: A Review. J. Pharm. Bioallied Sci. 2019, 11 (Suppl. 2), S126-S30. [CrossRef] [PubMed]

30. Pierce, G.F.; Mustoe, T.A.; Altrock, B.W.; Deuel, T.F.; Thomason, A. Role of platelet-derived growth factor in wound healing. J. Cell. Biochem. 1991, 45, 319-326. [CrossRef]

31. Melo, B.A.G.; Luzo, A.C.M.; Lana, J.; Santana, M.H.A. Centrifugation Conditions in the L-PRP Preparation Affect Soluble Factors Release and Mesenchymal Stem Cell Proliferation in Fibrin Nanofibers. Molecules 2019, 24, 2729. [CrossRef]

32. Liu, Z.; Jin, H.; Xie, Q.; Jiang, Z.; Guo, S.; Li, Y.; Zhang, B. Controlled Release Strategies for the Combination of Fresh and Lyophilized Platelet-Rich Fibrin on Bone Tissue Regeneration. BioMed Res. Int. 2019, 2019, 1-10. [CrossRef] [PubMed]

33. Tsay, R.C.; Vo, J.; Burke, A.; Eisig, S.B.; Lu, H.H.; Landesberg, R. Differential growth factor retention by platelet-rich plasma composites. J. Oral Maxillofac. Surg. 2005, 63, 521-528. [CrossRef] [PubMed]

34. Strong, D.M. Tissue banking, biovigilance and the notify library. Cell Tissue Bank. 2018, 19, 187-195. [CrossRef] [PubMed]

35. Han, S.K.; Kim, D.W.; Jeong, S.H.; Hong, Y.T.; Woo, H.S.; Kim, W.K.; Gottrup, F. Potential use of blood bank platelet concentrates to accelerate wound healing of diabetic ulcers. Ann. Plast. Surg. 2007, 59, 532-537. [CrossRef]

36. Jeong, S.H.; Han, S.K.; Kim, W.K. Treatment of diabetic foot ulcers using a blood bank platelet concentrate. Plast. Reconstr. Surg. 2010, 125, 944-952. [CrossRef]

37. Nather, A.; Mandy, F.S.Y.; Ning, T.; Kaiying, W. Tissue banking in Asia Pacific region: Past, present and future. Cell Tissue Bank. 2018, 19, 229-240. [CrossRef]

38. Ansarizadeh, M.; Mashayekhan, S.; Saadatmand, M. Fabrication, modeling and optimization of lyophilized advanced platelet rich fibrin in combination with collagen-chitosan as a guided bone regeneration membrane. Int. J. Biol. Macromol. 2019, 125, 383-391. [CrossRef]

39. Zhang, J.; Qi, X.; Luo, X.; Li, D.; Wang, H.; Li, T. Clinical and immunohistochemical performance of lyophilized platelet-rich fibrin (Ly-PRF) on tissue regeneration. Clin. Implant. Dent. Relat. Res. 2017, 19, 466-477. [CrossRef]

40. Sell, S.A.; Wolfe, P.S.; Ericksen, J.J.; Simpson, D.G.; Bowlin, G.L. Incorporating platelet-rich plasma into electrospun scaffolds for tissue engineering applications. Tissue Eng. Part. A 2011, 17, 2723-2737. [CrossRef]

41. Dohan Ehrenfest, D.M.; Pinto, N.R.; Pereda, A.; Jiménez, P.; Corso, M.D.; Kang, B.-S.; Nally, M.; Lanata, N.; Wang, H.-L.; Quirynen, $\mathrm{M}$. The impact of the centrifuge characteristics and centrifugation protocols on the cells, growth factors, and fibrin architecture of a leukocyte- and platelet-rich fibrin (L-PRF) clot and membrane. Platelets 2017, 29, 171-184. [CrossRef]

42. Wong, C.C.; Chen, C.H.; Chan, W.P.; Chiu, L.H.; Ho, W.P.; Hsieh, F.J.; Chen, Y.T.; Yang, T.L. Single-Stage Cartilage Repair Using Platelet-Rich Fibrin Scaffolds With Autologous Cartilaginous Grafts. Am. J. Sports Med. 2017, 45, 3128-3142. [CrossRef] [PubMed] 